\title{
Wake-up Radio-enabled Intermittently-powered Devices for Mesh Networking: A Power Analysis
}

\author{
Edward Longman Oktay Cetinkaya Mohammed El-Hajjar Geoff V. Merrett
}

\author{
Centre for IoT and Pervasive Systems \\ School of Electronics and Computer Science \\ University of Southampton, Southampton SO17 1BJ, UK \\ $\{$ el7g15, oc1y18, meh, gvm $\} @$ ecs.soton.ac.uk
}

\begin{abstract}
This paper analyzes the successful communication probability between two intermittently-powered nodes in a homogeneous energy harvesting $(\mathrm{EH})$ mesh network. Powering devices using EH can enable networks to operate indefinitely; however, with limited energy storage and in scarce EH conditions, nodes may only be intermittently-powered. This reduces the effectiveness of conventional networking techniques, where listening modes of radios deplete the storage too quickly, rendering nodes useless. This is particularly problematic in the deployment of mesh networks, where there is no provision of a high-power coordinator. To counter this, wake-up receivers (WuRxs) provide extended listening time without the need for a high power conventional radio, but with a cost to sensitivity. Therefore, listening time must be balanced with transmission and wake-to-receive cost, where if all the harvested energy is spent listening none remains to transmit, and vice versa. From stochastic analysis and simulation of the energy usage in mesh nodes, we obtain the optimum transmission load to maximize goodput, which is the rate of successful communications. We include the cost of each wake-up based on the network size in our goodput analysis. Simulations for a fixed number of homogeneous nodes verify this. Furthermore, we model and evaluate the energy consumption trade-off between transmit power and WuRx sensitivity to enable the maximum goodput.
\end{abstract}

Index Terms-Energy-harvesting, Batteryless, Mesh networking, Wireless communication, Throughput maximization, Wakeup radio

\section{INTRODUCTION}

The continued proliferation of Internet of Things (IoT)connected devices raises new challenges to ensure long lifetimes with scarce energy resources [1]. IoT devices are traditionally powered from batteries, which eventually deplete and have to be replaced. However, with the increasing scale of IoT networks, such maintenance will become impractical, and hence research efforts are focused on replacing batteries with energy harvesting (EH) sources, which convert ambient energy into electricity [2].

Although EH theoretically provides energy indefinitely, thus increasing longevity, most exploitable sources are unpredictable, and their output power varies by many orders of magnitude both temporally and spatially [3]. Furthermore, everevolving IoT applications necessitate operation under even the most severe EH conditions [4]. By adopting a small amount of energy storage (e.g. $10 \mathrm{~mJ}$ ) for communication, these devices

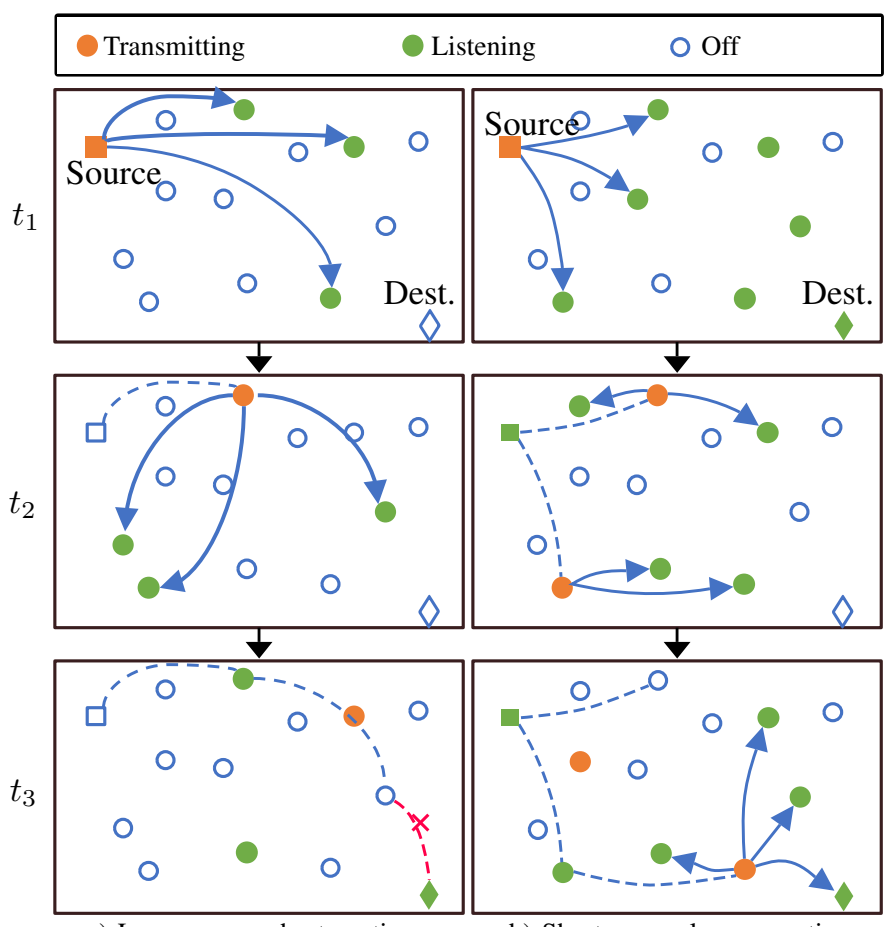

a) Large range, short on time

b) Short range, longer on time

Fig. 1: Comparison of intermittent homogeneous mesh configurations at consecutive periods, following a message from Source to Dest. for different range/power priorities. Consecutive periods $t_{1}, t_{2}, t_{3}$ show links within the period and dotted lines show previous links. Network a) has higher power receivers extending the range. Network b) has reduced range, lower power receivers which enables a longer listening time, leading to correct reception at $t_{3}$.

can operate intermittently where operation occurs with the available power [5]. The intermittency is because the output of the energy harvester cannot sustain device operation, so large tasks take place in consecutive power cycles [6].

For communication, intermittency causes forwarding and acknowledgment to take place across different power cycles to reception, as shown in Fig. 1 where hops take place in subsequent power cycles. When enough energy is harvested, the nodes can transmit messages including forwarding messages, and then receiving nodes can subsequently forward after they have harvested enough energy. However, since nodes 


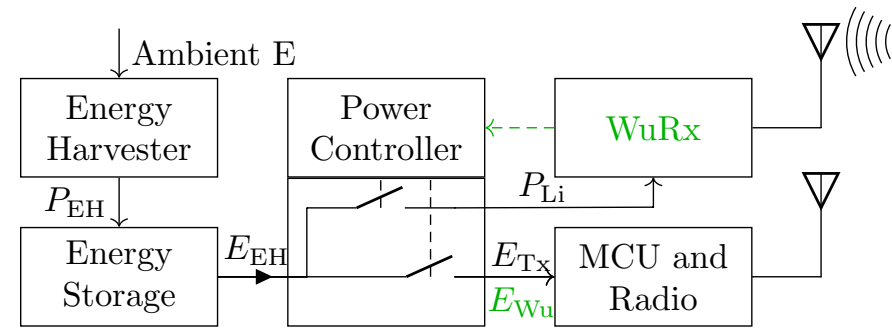

Fig. 2: Components of EH wireless sensor node and flow of energy.

are intermittent in Fig. 1a), despite the destination being immediately in range of the source, the data is not received because the destination is off and not listening. To increase the probability of receiving messages, methods to extend the listening capability are required.

Several methods are available to receive messages in scarce EH conditions by extending the listening time. Synchronous methods greatly reduce the radio on time, but frequent power outages in intermittent devices would lead to greatly increased resynchronization energy, so we only consider asynchronous approaches here. Previous efforts adopt wake-up receivers (WuRxs) in a star topology [7], [8] where energy-constrained edge devices are coordinated by a high-capability node. WuRxs can facilitate intermittency by providing a method of waiting to receive messages that consumes $\mu \mathrm{W}$ of stored energy [9]. However, having a high-capability coordinator may not be possible in some cases, e.g. aerial deployments to remote environments, disaster response networks, and geosensing [10], all requiring autonomous and flexible operation.

Given the advantage of extended listening time with WuRx, but the need for autonomy of operation in IoT networks, this paper focuses on communication in intermittent mesh networks-backed by WuRxs as shown in Fig. 1, which is an area unexplored by existing research. Previously, EconCast [11] considers intermittently connected mesh nodes and uses a Markov model to determine the broadcast throughput. However, it does not consider using WuRxs, nor the additional cost of each wake-up, with additional receiving and processing consumption, rather it only considers radios that have constant power consumption during the entire receive time (including listening). Whilst simulation results in EconCast are useful, an analytical form is needed to investigate a broader range of parameters; For example, using real power consumption and sensitivity data from commercial radios.

In order to enable wake-ups in a mesh topology, we consider nodes equipped with WuRxs and primary radios, shown in Fig. 2. Nodes in transmitting states can pass messages to nodes in the listening state, incurring a wake-up cost, but nodes in the off state cannot receive or send. Considering the radio power consumption of transmitting, Fig. 1a) shows that while using conventional higher power receivers increases the range it does not compensate for the resultant lower proportion of nodes listening. With subsequent forwarding, despite the destination listening in $t_{3}$, no transmitting neighbors have received the message from the source. Secondly in b), using WuRx to re- duce the listening power, which also reduces range, but means there are more nodes listening, the information successfully reaches the destination after 3 periods. This demonstrates how WuRx could improve the network performance, despite their downsides. Therefore, we observe the potential for increased hops to outperform high sensitivity listening.

Since the allocation of harvested energy for transmitting or listening and receiving affects what state each node is in, we analyze the time spent in these states to determine the performance under various transmission rates and radio power consumptions, leading to the following contributions:

- We model the energy consumption in $\mathrm{EH}$, but intermittently-powered, WuRx-enabled nodes, which is used to maximize the goodput as well as analyze the relationship between the goodput and transmissions rate.

- We analyze the effect of wake-ups and network density on the optimum transmission rate and maximum goodput, where we incorporate stochastic analysis for a Poisson point process (PPP) distributed network for our analysis.

- We demonstrate, using a model derived from commercial radio transmitters and typical WuRxs, the best configurations for different network densities, to achieve the highest goodput.

Our mathematical model shows that, without the impact of wake-ups, allocating energy equally for transmission and for listening maximizes the goodput (confirming simulation results of EconCast [11]). However, when the wake-up impact is also considered, the proportion of energy used for transmitting should be reduced. When including a model of primary radio and WuRx hardware, we analyze the effect of receiver sensitivity on the goodput across a range of node densities.

The rest of this paper is organized as follows. In Section II we define how nodes operate, are distributed and the conditions of our analysis. Following this, in Section III we use the energy consumption of each of the node components to formulate equations describing the goodput for varying loads. We expand this analysis to incorporate the effect of wake-ups and a variety of real hardware. We then show the results of our analysis, in Section IV, partially validated by simulations, and discuss how they can be used to determine network performance. The paper is concluded by noting that the hardware chosen is important to achieve good performance and what future work is required to realize intermittently powered networks.

\section{SySTEM MODEL}

In this paper, we first consider a node in a WuRx-enabled mesh network. The node uses ambient EH to charge a small energy store and power a threshold-based power controller, as shown in Fig. 2. When a node's supply is restored, termed an EH event, it can turn on and broadcast a wake-up signal followed by data transmission to its vicinity using a network ID for addressing [8], which consumes $E_{\mathrm{Tx}}$. The remaining energy in the power cycle is used for listening and receiving, consuming power $P_{\mathrm{Li}}$, as shown in Fig. 2. The network nodes are spatially distributed according to a PPP and they all operate 
in the same three state manner, as shown in Fig. 1. Listening nodes in the vicinity when a node broadcasts a wake-up can receive the transmission. The wake-up ${ }^{1}$ at the listening nodes shown by the dotted line in Fig. 2 depletes the remaining energy by $E_{\mathrm{Wu}}$, and shortens the subsequent listening period.

We consider scarce EH conditions, meaning the data sent cannot be immediately acknowledged or forwarded, for two reasons: 1) the relay node might use all of its energy during reception, and 2) the next hop might still be in the off state, as shown in Fig. 1. Additionally, the number of communicating nodes, i.e. listening or transmitting, is significantly lower than the off nodes.

In order to maximize the performance of the network, nodes can adjust their transmission rate. Increasing the transmission rate may increase wake-ups at other nodes, but simultaneously decreases listening time at the transmitting node. Therefore the transmission rate and corresponding energy allocated for communication events has to be optimized for the maximum performance. For a mesh network, all nodes act to forward data around a network, therefore, in this paper we analyze energy consumption of one node where the neighbors experience the same average load and ambient EH.

The conditions/assumptions are summarized below:

(i) Nodes do not harvest enough to continuously listen.

(ii) EH events are uncorrelated and distributed as a PPP.

(iii) A node's energy storage is large enough to store multiple $\mathrm{EH}$ events in short succession to ensure independence of the operation of each event.

(iv) If a wake-up is successful, the subsequent data communication will also be successful, since the main radio has much higher sensitivity than the WuRx.

(v) The nodes are homogeneous, all having the same hardware and transmit/receive a similar number of packets.

Based on this network EH and node energy consumption model for intermittent devices, we analyze the performance using the throughput over a two node link in the network.

\section{COMMUNICATIONS PROBABILITY ANALYSIS}

For the communication analysis, we consider the link between two nodes in a mesh network of node density $\Lambda$. We consider the energy used for each communication event, i.e. listen, transmit and receive, to determine the probability of transmitting at each EH event, $\mathrm{p}(T x)$, and the probability of listening at any moment, $\mathrm{p}(L i)$. These probabilities lead to the derivation of the goodput.

To analyze our system model, we quantify the harvested energy and energy consumption (shown in Fig. 2), and communication parameters as follows. The energy harvester provides an average power, $P_{\mathrm{EH}}$ which is available to the node in discrete units, $E_{\mathrm{EH}}$, that are termed energy bursts, equivalent to a threshold-based on/off system. Furthermore, we define the energy arrival rate as $\lambda_{\mathrm{EH}}=\frac{1}{\tau_{\mathrm{EH}}}$, so $P_{\mathrm{EH}}=\lambda_{\mathrm{EH}} E_{\mathrm{EH}}$. The considered node has a primary radio and consumes $E_{\mathrm{Tx}}$

\footnotetext{
${ }^{1}$ Wake-up throughout this paper refers to received RF wake-up and not turning on when the energy storage is full, which is referred to as EH events.
}

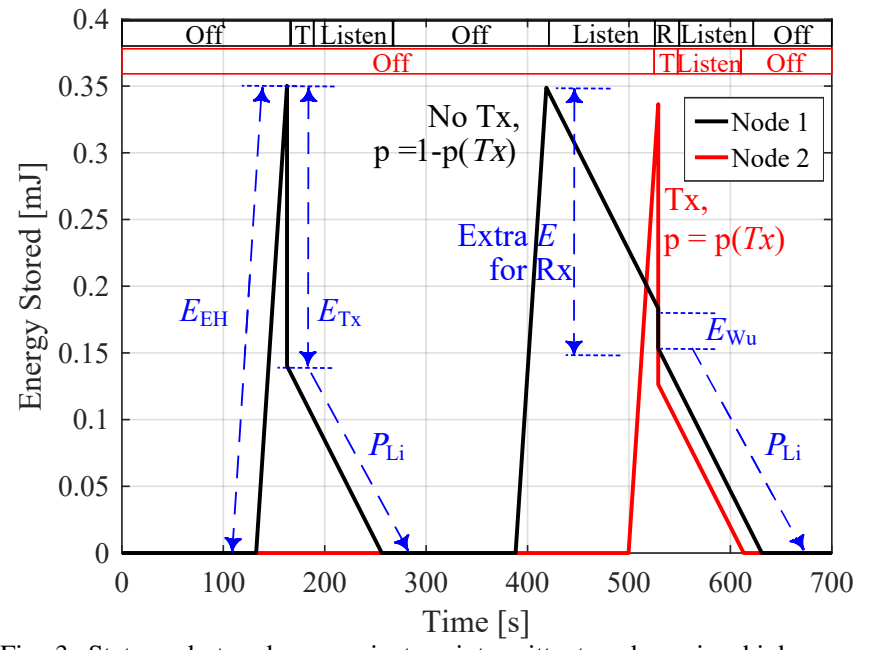

Fig. 3: State and stored energy in two intermittent nodes using high power wake-up transmissions to a WuRx. The stored energy of Node 1 is annotated in blue, and $\mathrm{T}$ and $\mathrm{R}$ are Transmission and Reception events respectively.

to transmit one wake-up signal and packet. The node has a WuRx with a fixed receive sensitivity, $T$, which consumes $P_{\mathrm{Li}}$ for listening. The node primary radio is used to receive the data after a wake-up, consuming $E_{\mathrm{Wu}}$, shown for Node 1 in Fig. 3.

The nodes operate as follows, when the harvested energy, $E_{\mathrm{EH}}$, is higher than the transmit energy, $E_{\mathrm{Tx}}$, the node transmits a packet from its queue, $Q$, depending on a load limiting probability, $\mathrm{p}(T x)$, to optimize the network performance. The remaining harvested energy after transmission, $E_{\mathrm{Rx}}$, can be used to listen for wake-ups and receiving. Fig. 3 shows this operation for a wasted transmission by Node 1 followed by a successful transmission by Node 2. Node 1 listens and wakes up when the WuRx receives a signal $I$ greater than $T$. The node transmit operation is limited by $\mathrm{p}(T x)$, which is a function of $E_{\mathrm{EH}}>E_{\mathrm{Tx}},|Q|>0$, and a retransmission probability. The probability of a node being able to listen for wake-ups is denoted by $\mathrm{p}(L i)$.

\section{A. Energy consumption analysis}

Here, we consider the consumption of $E_{\mathrm{EH}}$ following one $\mathrm{EH}$ event, based on (ii) and (iii) given in Section II. $E_{\mathrm{EH}}$, is used for transmitting in $\mathrm{p}(T x)$ of cases. The remaining energy, $E_{\mathrm{Rx}}$, is consumed for two things: $E_{\mathrm{Li}}$ for powering the WuRx for listening and $E_{\mathrm{Wu}}$ for each wake-up period at average active power $P_{\mathrm{Li}}$ and $P_{\mathrm{Wu}}$, respectively. Once all $E_{\mathrm{EH}}$ is used, the node turns off and is unable to receive, such as at $250 \mathrm{~s}$ and $610 \mathrm{~s}$ in Fig. 3, till the next energy burst.

For wake-ups transmitted with probability $\mathrm{p}(\mathrm{Wu}), P_{\mathrm{Wu}}$ is only consumed if the receiver is listening, like at $525 \mathrm{~s}$ in Fig. 3. This gives the expected time spent in wake-up per EH event, $\mathrm{p}(L i \cap W u) \tau_{\mathrm{EH}}$, which is equal to $\mathrm{p}(\mathrm{Wu}) \mathrm{p}(L i) \tau_{\mathrm{EH}}$ in consideration of (ii). From this, the average wake-up energy consumption per $\mathrm{EH}$ event can be calculated as $\mathrm{p}(\mathrm{Wu}) \mathrm{p}(L i) P_{\mathrm{Wu}} \tau_{\mathrm{EH}}$. Hence the $E_{\mathrm{EH}}$ generated is equal to the consumption as follows:

$$
E_{\mathrm{EH}}=\mathrm{p}(T x) E_{\mathrm{Tx}}+E_{\mathrm{Li}}+\mathrm{p}(\mathrm{Wu}) \mathrm{p}(L i) P_{\mathrm{Wu}} \tau_{\mathrm{EH}} .
$$


Following this, to find $\mathrm{p}(L i)$, we derive the average $E_{\mathrm{Li}}$ :

$$
E_{\mathrm{Li}}=E_{\mathrm{EH}}-\mathrm{p}(T x) E_{\mathrm{Tx}}-\mathrm{p}(\mathrm{Wu}) \mathrm{p}(L i) P_{\mathrm{Wu}} \tau_{\mathrm{EH}} .
$$

Given the energy arrival rate $\left(\lambda_{\mathrm{EH}}\right) \times E_{\mathrm{Li}}$ and constant $P_{\mathrm{Li}}$, we calculate $\mathrm{p}(L i)=\lambda_{\mathrm{EH}} E_{\mathrm{Li}} / P_{\mathrm{Li}}$, which expands to

$$
\mathrm{p}(L i)=\frac{\left[\lambda_{\mathrm{EH}} E_{\mathrm{EH}}-\lambda_{\mathrm{EH}} \mathrm{p}(T x) E_{\mathrm{Tx}}-\mathrm{p}(\mathrm{Wu}) \mathrm{p}(L i) P_{\mathrm{Wu}}\right]}{P_{\mathrm{Li}}},
$$

and by rearranging, we get

$$
\mathrm{p}(L i)\left[1+\frac{P_{\mathrm{Wu}} \mathrm{p}(\mathrm{Wu})}{P_{\mathrm{Li}}}\right]=\frac{\lambda_{\mathrm{EH}}}{P_{\mathrm{Li}}}\left[E_{\mathrm{EH}}-\mathrm{p}(T x) E_{\mathrm{Tx}}\right] .
$$

To derive the goodput we can substitute the transmission rate, $R=\lambda_{\mathrm{EH}} \mathrm{p}(T x)$. The transmissions at rate $R$ are received in $\mathrm{p}(L i)$ cases, and we define the goodput as $G=\mathrm{p}(L i) R$. By multiplying both sides of (3) with $R$ and using $G$ accordingly, we obtain the analytical relationship between $G$ and $R$ :

$$
G\left[1+\frac{P_{\mathrm{Wu}} \mathrm{p}(\mathrm{Wu})}{P_{\mathrm{Li}}}\right]=\frac{1}{P_{\mathrm{Li}}}\left[P_{\mathrm{EH}} R-E_{\mathrm{Tx}} R^{2}\right] .
$$

\section{B. Wake-up probability and impact on goodput}

Since the node is part of a network, described by density $\Lambda$, it is subject to wake-up events from multiple transmitting nodes, like in Fig. 1b) at $t_{3}$. Using the received signal strength, $I$, for a WuRx with sensitivity $T, \mathrm{p}(\mathrm{Wu})$ is defined as $\mathrm{p}(I \geq$ $T)$. When $\Lambda$ increases, $\mathrm{p}(I \geq T)$ increases and using PPP analysis from Kouzayha [12], $\mathrm{p}(\mathrm{Wu})$ can be modeled based on $T$ as:

$$
\begin{aligned}
\mathrm{p}(\mathrm{Wu})=\int_{0}^{\infty} \frac{1}{\pi u} \exp (-u T) & \exp \left(-\frac{2 \pi^{2} \delta \Lambda}{\alpha \tan \left(\frac{2 \pi}{\alpha}\right)}(\eta P u)^{\frac{2}{\alpha}}\right) \\
& \times \sin \left(\frac{2 \pi^{2} \delta \Lambda}{\alpha}(\eta P u)^{\frac{2}{\alpha}}\right) d u
\end{aligned}
$$

$P$ is the transmission power, $T$ is the receiver sensitivity, $\eta$ the antenna efficiency, and $\alpha$ is the path loss exponent. $\delta \Lambda$ is the transmitter density, where $\delta$ is the proportion of time nodes spend transmitting wake-ups, calculated from the beacon length and rate. The $\mathrm{p}(\mathrm{Wu})$ in (5) can be used in (4) to calculate the maximum $G$ based on $\Lambda$ as well as the channel and radio parameters.

This then allows for the effect of wake-ups from the mesh network to be included in the goodput for the analyzed link.

\section{Optimum transmission/listening energy consumption ratio}

Inspecting (4) and (5) reveals the interdependence of the primary radio and WuRx consumption with the output power and sensitivity. In a WuRx-enabled mesh network, increased WuRx sensitivity corresponds to increased $P_{\mathrm{Li}}$ [13], while decreased sensitivity increases $E_{\mathrm{Tx}}$ for the same range [14], shown in Fig. 4. Based on real radio characteristics, we analyze the energy used for a fixed-loss link in a network operating in the $868 \mathrm{MHz}$ Band. We consider the trade-off between transmission energy requirements and receiver listening capability.

In Fig. 4 we show the consumption model for the transmitter and WuRx with a $-50 \mathrm{~dB}$ link and the energy required for an

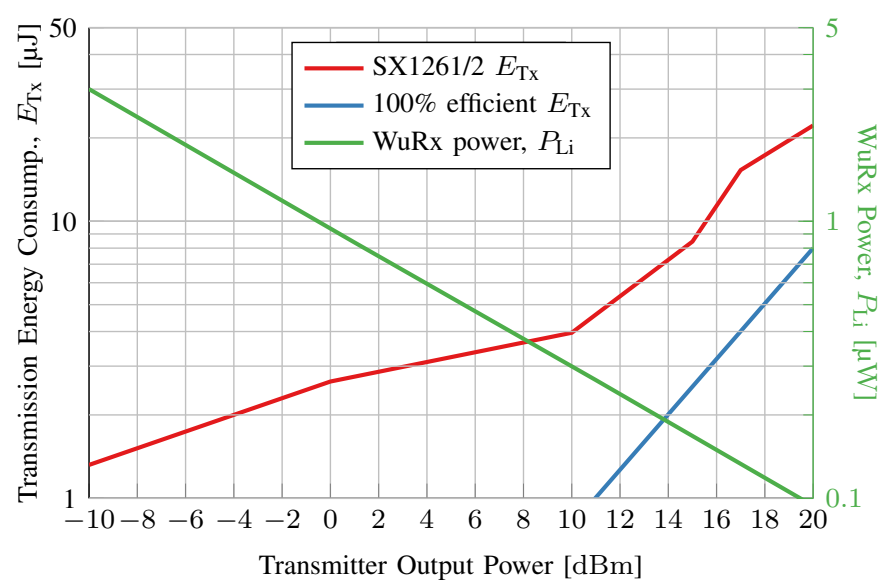

Fig. 4: Model of energy consumption per transmission (including wake-up beacon), and the minimum consumption of a WuRx which can receive it after $-50 \mathrm{~dB}$ link loss [13], [15].

$80 \mathrm{~ms}$ packet. The model is based on the Semtech SX1261/2 [15]. Due to the lower efficiency of the power amplifiers (PAs) at lower power the increase in range is approximately proportional to power consumed for powers up to $10 \mathrm{dBm}$. The WuRx model is formed by the relationship between listening power consumption and sensitivity $(T)[13]$ as:

$$
10 \log \left(P_{\mathrm{Li}}\right)=-20 \log (T)+60 .
$$

By using the relationship in Fig. 4, the $G$ over a fixedloss link can be analyzed. Initially, only the listening cost is considered, and not the additional wake-up cost $\left(P_{\mathrm{Wu}}=0\right)$, so the solution to (4) gives

$$
G_{\max }=\frac{P_{\mathrm{EH}}^{2}}{2 P_{\mathrm{Li}} E_{\mathrm{Tx}}} .
$$

The maximum including the effect of wake-ups $\left(P_{\mathrm{Wu}}>0\right)$ can be solved numerically, e.g. using the Newton-Raphson method, with $G_{\max }$ as a starting estimate. By using the parameters from Fig. 4 in (4), the best transmitter power and WuRx configuration can be found.

\section{RESUlTS}

Using the analysis in the previous section, we present the variation of goodput, $G$, for the varying transmission load, $R$, different node densities, $\Lambda$, and primary radio and WuRx combinations. The overhead of wake-ups from a fixed number of neighbors is considered and validated with MATLAB simulations. Finally, the results of the analysis of realizable transmitters and receivers is presented.

The simulation consists of models of the nodes with components consuming power as specified in Table I. The nodes operate as specified in Section II based on their value of stored energy. Each component consumes energy according to the operation and the resulting energy traces are shown in Fig. 3. Information packets are generated at a fixed rate forming the load. For the multi-node case all nodes receive energy and packets independently. Also, all nodes are within range of each other and a perfect channel model is assumed. The simulations are run for $10 \mathrm{Ms}$ equivalent to 115 days of operation. 


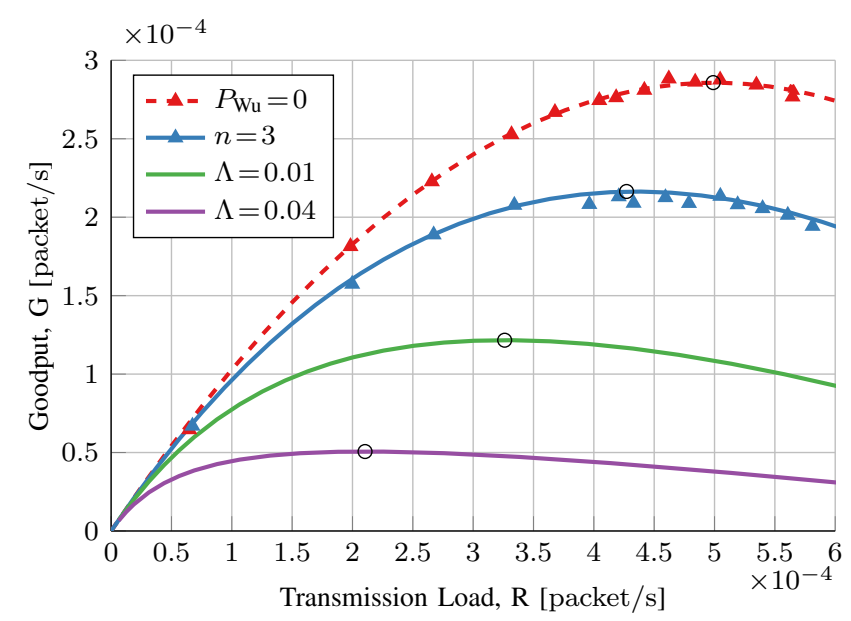

Fig. 5: Transmission load and Wake-up impact on goodput. Simulation results to validate the analytical solution marked by $\boldsymbol{\Lambda}$ and maximum goodput by $\circ$.

\section{A. Optimum load for maximum goodput with a fixed number of neighbors}

Evaluating (4), when the node operates intermittently, shows increasing $G$ as $R$ is increased until $G_{\max }$, beyond which $G$ decreases. Fig. 5 shows that, when the overhead of each wakeup is 0 , the maximum $G$ is achieved at $R_{\mathrm{opt}}=P_{\mathrm{EH}} /\left(2 P_{\mathrm{Li}} E_{\mathrm{Tx}}\right)$, when energy is split equally for transmitting and receiving. This result matches other authors simulation that does not consider the cost of waking up [11]. Above $R_{\text {opt }}$, the reduction in listening energy and time outweighs the extra transmissions.

We also include $P_{\mathrm{Wu}}$ for three neighboring nodes, $n=3$, when evaluating (4). This is shown in Fig. 5 where both the maximum $G$ and $R_{\text {opt }}$ are reduced as energy is instead used for the wake-ups. We use $E_{\mathrm{Wu}}$ averaged across the transmission time in Section III-C for our value of $P_{\mathrm{Wu}}$.

Both zero overhead wake-ups and the fixed number of neighbors models have been verified with simulations with the parameters provided in Table I. For a fixed number of neighbors, it is assumed that there is a perfect channel and they are all within range of each other. We simulate the energy level over time (as in Fig. 3) for EH events and radio consumption to validate the analytical function of $G$ in (4).

\section{B. Impact of transmitter density on goodput}

Beyond a fixed number of neighbors, we analyze the effect of $\Lambda$ and transmission power. When $\mathrm{p}(\mathrm{Wu})$ in (5) is included in the optimum load calculations (4), increasing $\mathrm{p}(\mathrm{Wu})$ reduces

TABLE I: Parameters used in analysis and simulations.

\begin{tabular}{rcl}
\hline Symbol & Parameter & Value \\
\hline$P_{\mathrm{Li}}$ & WuRx listening consump. & $0.35 \mu \mathrm{W}$ \\
$E_{\mathrm{Wu}}$ & Wake-up energy consump. & $80 \mu \mathrm{J}$ or 0 \\
$E_{\mathrm{Tx}}$ & Transmission energy consump. & $400 \mu \mathrm{J}$ \\
$\lambda_{\mathrm{EH}}$ & Harvesting event rate & $6.66 \times 10^{-4} \mathrm{~s}^{-1}$ \\
$E_{\mathrm{EH}}$ & Harvesting burst energy & $\mathcal{N}\left(\mu=600 \mu \mathrm{J}, \sigma^{2}=40 \mu \mathrm{J}^{2}\right)$ \\
$P_{\mathrm{EH}}$ & Average harvested power & $0.4 \mu \mathrm{W}$ \\
$\mathrm{R}$ & Transmission rate & 0.66 to $6 \times 10^{-4} \mathrm{~s}^{-1}$ \\
$\Lambda$ & Node density & 0 to $5 \times 10^{-2} \mathrm{~m}^{-2}$ \\
$\alpha$ & Path loss & 2.6 \\
$\frac{\eta P}{T}$ & Link attenuation & $35 \mathrm{~dB}$ \\
\hline
\end{tabular}



Fig. 6: $\mathrm{p}(\mathrm{Wu})$ as a function of $\frac{\eta P}{T}, \alpha$ and $\delta \Lambda$, and baseline parameters of $\frac{\eta P}{T}=35 \mathrm{~dB}$ and $\alpha=2.6$

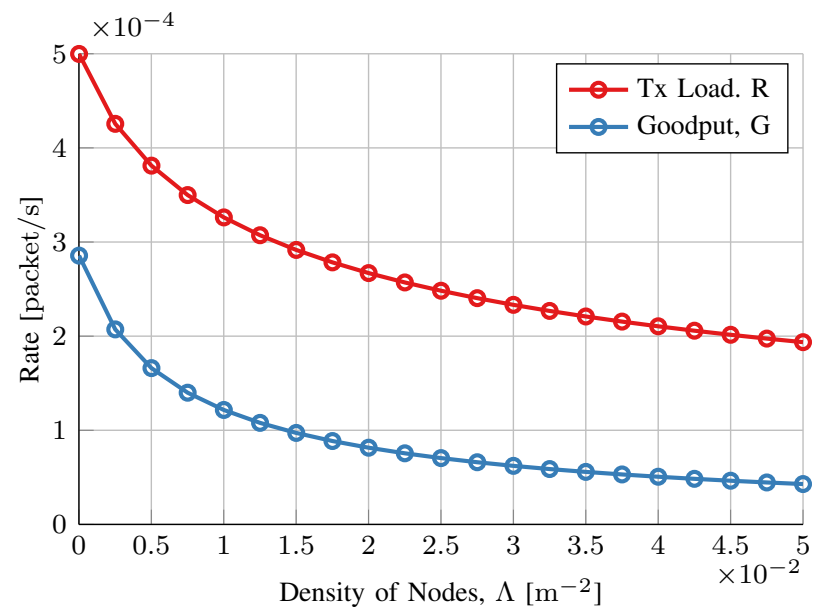

Fig. 7: Optimum load and corresponding $G$ for a mesh network of density $\Lambda$.

$G_{\max }$ because the energy is used to handle wake-ups. Since the impact of a wake-up increases $E_{\mathrm{Rx}}$, the load must be decreased to ensure sufficient $\mathrm{p}(L i)$, as in Fig. 5. If the load is not decreased for $\Lambda=0.01, G$ is then $10 \%$ below $G_{\max }$. This shows the importance of adding wake-up cost consideration that previous work [11] doesn't consider. The variation in $\mathrm{p}(\mathrm{Wu})$ from (5) is shown in Fig. 6 where increasing the ratio of $\frac{\eta P}{T}$ increases (proportionally to the power) the $\mathrm{p}(\mathrm{Wu})$, and increasing $\alpha$ decreases $\mathrm{p}(\mathrm{Wu})$.

When the transmission power required to reach the average separation between two nodes is included, increased density causes more energy to be spent processing wake-ups, so less remains for listening, as shown in Fig. 5 and Fig. 7. As the density increases the proportion of failed wake-ups increases, i.e. $45 \%$ at $\Lambda=0$ but $90 \%$ fail at $\Lambda=0.05$. This corresponds to a higher proportion of retransmissions.

\section{Choosing Tx and WuRx combinations at different densities}

The model for $\mathrm{p}(\mathrm{Wu})$ and the transmitter/WuRx consumptions can be combined with (4) to find the maximum $G$ for varying WuRx sensitivity and average density as in Section III-C. This is so that the best hardware combination can be 




Fig. 8: $G_{\max }$ when varying the density and required link budget. Transmitter consumption, $E_{\mathrm{Tx}}$, at highest $G$ annotated and Table I value circled.

chosen for a particular deployment. We specify that each node has a range of $2 \times$ average nearest neighbor distance, chosen to ensure $99 \%$ of nodes are within range of at least two others. Therefore the link loss affecting the required transmit power is derived from the Friis equation at each density.

Fig. 8 shows the benefit of using high power transmissions across a range of densities to achieve the highest goodput. Consequently, for dense networks, it is beneficial to use increased transmit power so long as $E_{\mathrm{Li}}$ reductions outstrip the increase in $E_{\mathrm{Tx}}$. For the $\mathrm{SX} 1261 / 2$ radio this means transmit consumption stays relatively constant around $400 \mu \mathrm{J}$. The WuRx can then be chosen based on the expected link loss.

The maximum $G$ is a consequence of the $E_{\mathrm{Tx}}$ and $P_{\mathrm{Li}}$ tradeoff, where after the inflection point at $P=10 \mathrm{dBm}$ shown in Fig. 4 there is reduced efficiency improvements as transmission power increases. Therefore, at higher transmission power, $E_{\mathrm{Tx}}$ increases faster than the decrease in $P_{\mathrm{Li}}$, so the best transmitter power is where $E_{\mathrm{Tx}}$ begins to grow faster than $E_{\mathrm{Li}}$ falls, at each link loss. On the other hand, when $E_{\mathrm{Wu}}$ is included, the benefit of very low $P_{\mathrm{Li}}$ is reduced, since $E_{\mathrm{Rx}}$ remains constant, however, the effect of this is minimal and the transmitter radio characteristics dominate.

The model was verified with the same simulation as Section IV-A where the radio parameters were chosen to be close to the highest goodput at $\Lambda=0.05$, as specified in Table I. The result is circled in Fig. 8. This model can be adapted for other hardware to understand both the achievable goodput in the intermittent network and also to choose primary radio and WuRx appropriately to improve network performance.

\section{CONCLUSIONS AND Future WORK}

This paper presents a power analysis of trade-offs in intermittently-powered communications, to show how goodput is affected by $\mathrm{EH}$, listening/transmitting energy consumption, and wake-ups. The analysis shows that mesh networking with intermittently-powered devices is achievable, but radio hardware and transmission rate greatly affect the performance. While there are limitations on goodput due to the intermittency, mesh networking of these devices allows for networks to be implemented in scenarios where EH previously is insufficient to sustain node operation. These results will help with the design of networks in the selection of device hardware, ensuring $\mathrm{EH}$ power is appropriate for the desired communication rate. They also form a benchmark for future intermittently-powered communication methods.

Further work is needed on MAC layer techniques that can enable communication when energy harvesting is too scarce to listen. Also, techniques to enable acknowledgment, sequencing, and delay-tolerant routing across both MAC and network layers are required for intermittent mesh networking solutions to be realized.

\section{ACKNOWLEDGMENT}

This work was supported in part by the UK EPSRC under $\mathrm{EP} / \mathrm{P} 010164 / 1$. The simulation data and figure generation scripts are available at https://doi.org/10.5258/SOTON/D1643.

\section{REFERENCES}

[1] O. B. Akan et al., "Internet of hybrid energy harvesting things," IEEE Internet Things J., vol. 5, no. 2, pp. 736-746, Apr. 2017.

[2] O. Cetinkaya et al., "Energy-neutral wireless-powered networks," IEEE Wireless Commun. Lett., vol. 8, no. 5, pp. 1373-1376, Oct. 2019.

[3] M. Gorlatova, A. Wallwater, and G. Zussman, "Networking low-power energy harvesting devices: Measurements and algorithms," IEEE Trans. Mobile Comput., vol. 12, no. 9, p. 1853-1865, Sep. 2013.

[4] P. Gleonec et al., "Architecture exploration of multi-source energy harvester for IoT nodes," in 2016 IEEE Online Conference on Green Communications, Nov. 2016, pp. 27-32.

[5] K. S. Yildirim and P. Pawelczak, "On distributed sensor fusion in batteryless intermittent networks," in 15th Int. Conf. on Distrib. Comput. in Sensor Systems, May 2019, pp. 495-501.

[6] S. T. Sliper et al., "Energy-driven computing," Philosophical Transactions of the Royal Society A, vol. 378, p. 20190158, 2019.

[7] F. A. Aoudia et al., "Leveraging energy harvesting and wake-up receivers for long-term wireless sensor networks," Sensors, vol. 18, no. 5, 2018.

[8] — - "SNW-MAC: An asynchronous protocol leveraging wake-up receivers for data gathering in star networks," in Sensor Systems and Software. Springer International Publishing, 2017, pp. 3-14.

[9] D. Spenza et al., "Beyond duty cycling: Wake-up radio with selective awakenings for long-lived wireless sensing systems," in 2015 IEEE Conf. on Computer Comms., pp. 522-530, ISSN: 0743-166X.

[10] B. Rashid and M. H. Rehmani, "Applications of wireless sensor networks for urban areas: A survey," Journal of network and computer applications, vol. 60, pp. 192-219, 2016.

[11] T. Chen et al., "Maximizing broadcast throughput under ultra-low-power constraints," IEEE/ACM Trans. Netw., vol. 26, no. 2, pp. 779-792, Apr. 2018.

[12] N. Kouzayha et al., "Joint downlink/uplink rf wake-up solution for iot over cellular networks," IEEE Trans. Wireless Commun., vol. 17, no. 3 , pp. 1574-1588, Dec. 2017.

[13] D. D. Wentzloff, "Ultra low power radio survey." [Online]. Available: www.eecs.umich.edu/wics/low_power_radio_survey.html

[14] R. Su, T. Watteyne, and K. S. J. Pister, "Comparison between preamble sampling and wake-up receivers in wireless sensor networks," in 2010 IEEE Global Telecommunications Conf., Dec. 2010, pp. 1-5.

[15] Semtech, "Sx1261/2 long range, low power, sub-GHz RF transceiver," p. 17. [Online]. Available: www.semtech.com/products/wireless-rf/ lora-transceivers/sx1261 